Advances in Computational Sciences and Technology

ISSN 0973-6107 Volume 10, Number 5 (2017) pp. 885-894

(C) Research India Publications

https://dx.doi.org/10.37622/ACST/10.5.2017.885-894

\title{
Identifying Lump Size in Brain MRI using Hierarchical Mean Shift Algorithm
}

\author{
Sanjeeva Polepaka ${ }^{1}$, Dr. Ch. Srinivasa Rao², Dr. M. Chandra Mohan ${ }^{3}$ \\ ${ }^{1}$ Research Scholar JNTUH \& Associate Professor, Department of Computer Science \\ and Engineering, MREC (Autonomous), Secunderabad-500100, India.
}

${ }^{2}$ Professor, Department of Electronics and Communication Engineering, JNTUKCEV, Vizianagaram, India.

${ }^{3}$ Professor, Department of Computer Science \& Engineering, JNTUHCEH, JNTU

Hyderabad, India.

\begin{abstract}
At the present scenario, identifying the brain tumor in the early stage using Magnetic Resonance Imaging (MRI) has become a popular research area. In children, brain tumors are the cause of one-quarter of all cancer deaths. Nowadays, cancer has become a major issue in human life to forecast the cancer lumps in the human brain. There are so many image processing algorithms to deal with the tumor identification. Out of bunch-of-algorithms, an intelligent algorithm is presented in this paper to identify the chunk size using Hierarchical Mean shift algorithm. Experimental results showed that the proposed algorithm had detected the tumors accurately when compared to Fuzzy C-Means (FCM) approach based tumor detection.
\end{abstract}

\section{INTRODUCTION}

In medical community medical imaging has been enhanced rapidly. Recent technologies are developed for observing, analyzing and treating the patients. These have the accessibility of appropriate indicators for quick progress. Structural arrangements can be successfully imaged today with Magnetic resonance imaging (MRI), X-Ray, Computed Tomography (CT), Ultra Sound and Optical Imaging methods. Physiological structures regarding metabolisms and functions can be further obtained through Positron Emission Tomography (PET), nuclear medicine, Single Photon Emission Computed Tomography (SPECT) and other derived protocols 
of MRI such as diffusion sensor MRI. Unlike CT scans and conventional x-ray examinations, MRI does not utilize on ionizing radiation. While analyzing brain morphology, segmenting tissues into three parts, namely, Gray Matter, White Matter and Cerebro Spiral Fluid, is a significant step.

Most of the medical image analysis initiates with the segmentation of images process because this stage plays a vital role in identifying the distinct regions of the image. Segmentation is a process of dividing the image into different regions and carried out by grouping the neighborhood pixels based on a predefined similarity criterion. While segmenting the parameters of the image, adaptive mean-shift framework [1] is involved. Hence the segmentation process is much accurate.

Non-symmetric distributions such as skew normal distribution are well suited for analyzing the medical data as it has multivariate distribution and the Gaussian distribution is a particular case of skew-normal distribution. Hence, to obtain better results, the whole image is characterized with a finite skew normal distribution mixture model. Foremost step in mixture models is to get the number of "k" regions (components), the formerly known part number in Expectation-Maximization (EM) algorithm [2] for image segmentation in parameter initialization. Bayesian priori, a random sampling of the mixture data, is commonly used in the parametric initialization method. Computational complexity is increased with increase in size, and as size decrease's, regions are not sampled. Thus, the accurate result might not occur. To analyze the image, an algorithm is developed to segment the acquired image based on finite skew normal mixture model with k-means algorithm. In this method, the number of components is identified using a k-means algorithm, and EM algorithm takes the parameter initialization process. The segmentation performance is evaluated by obtaining the quality metrics like Jacquard coefficient, Variation of Information, Volume Similarity coefficients, Probabilistic Rand Index and Global Consistency Error, by applying on different brain images.

This paper follows the following organization. Section 2 deals with the literature review. Section 3 is concerned with proposed methodology. The implementation and results are discussed in sections 4 and 5 respectively. Finally, section 6 draws the conclusion.

\section{EXISTING METHODS}

This section deals with the existing methods of brain tumor segmentation methods.

Kim [7] proposed a new method to detect brain tumor on the Magnetic Resonance Images (MRI) and enabled the efficient transfer of image files between the Healthcare Providers using Internet. The Conventional K-Means Clustering technique was modified in this method. The initial segmentation was done through Mean Shift segmentation. After the conversion of the segmented image to grayscale, Watershed algorithm detected the brain tumor. The comparative study of the result with the conventional FCM Clustering and K-Means Clustering revealed an excellent outcome. Kim's modified method yielded 0.914052 and 0.995641 in the precision and 
recall results. Even though this approach performed well with the reduced image size (instead of original size) without affecting the features, this method was examined on ten images collected from Google search engine. Further, this method was not evaluated on standard datasets.

Esmail Hassan [8] proposed a GUI based Matlab interface to detect brain tumor from MRI images. Initially, the image was filtered with Prewitt Horizontal Edge emphasizing filter. The brain tumor was recognized with "Watershed pixels". The authors claimed that GUI Matlab Guide had enhanced the segmentation process to detect the tumor by using various combinations of filters. Though this method used Sobel Edge Mask method for filtering, the possibility of using other combination of filters and image processing techniques are left unexplained.

Pavel Dvorak [9] focused on Automatic Detection of Brain Tumor with T2-Weighted Magnetic resonance images. The brain's left-right symmetry property was utilized to detect the tumor in the unhealthy (affected) brain. Larger the variations in the features confirm the presence of a tumor.

Shanmuga Priya [10] adopted Support Vector Machines(SVMs) to segment the brain tumors in Computed Tomography images by comparing two Kernel functions of SVM. The author used 50 feature vectors as input to the SVM model for an image. The polynomial and RBF Kernel were applied on the segmented tumor image and compared with the ground truth values derived from the radiologist. Authors concluded that RBF Kernel method outperformed the polynomial based Kernel method. The segmentation accuracy may be improvised by optimizing the SVM parameters, such as box constraint and RBF sigma values.

Ramaraju et al [11] proposed a method for brain tumor stage classification and segmentation process through Probabilistic Neural Network techniques. While the tumor stage classification was done through the neural network, the detection of the tumor was carried out by K-Means Clustering algorithm followed by CAD (Computer Aided Design). This proposed method was tested on a set of images acquired from different patients. When this method was tested on a set of images, results revealed that the classification, segmentation and tumor detection processes were done easily. Though this approach detected the tumor successfully, it failed to estimate the growth of tumor cells in a patient.

S. Ghanavathi [12] presented an automatic tumor detection algorithm using multimodal MRI. The most discriminative features were selected using the AdaBoost classifier to segment the tumor region. This approach was assessed on five sets of Gad MR, T1, T2 images with different size, shape, location and contrast generated by TumorSim Software. The experimental result shows 100\% successful tumor detection with an average accuracy of about $90 \%$. This algorithm has improved segmentation accuracy when considering shape deformation feature. This proposed algorithm failed to monitor the tumor growth and its response to the treatment over time. It failed to evaluate the surgical outcome too. 
Ahmed Kharrat et al [13] proposed a method to detect brain tumor in medical images or cerebral MRI images. Initially, the input image's quality was improved and the tumor detection was done using mathematical morphology, Wavelet Transform and finally the K-Means algorithm. When the images were enhanced, the Contrast Improvement Index (CII) factor was improved in Mathematic Morphology rather than CLACHE algorithm and Beghdadi algorithm. After the image decomposition, the tumor was extracted by using K-Means method. When the proposed method was compared with the performance of expert's result, the PSNR and MASD values found to be in the acceptable ranges of about 21.6272 and 17.7768. Though this method reduced the steps for extraction, using image enhancement failed to classify the tumor as "benign" or "malignant". This method highlights only pathological features but not other factors.

Dimple [14] suggested Brain Tumor detection from MRI images by employing Skull Stripping algorithm. For effective Skull Stripping, the MRI images were segmented via Self-Organizing Map and Neural Network (NN), preceded by the extraction of features by Stationary Wavelet Transform. Dimple worked on BRAINX medical images as a dataset. The efficacy of the Skull Stripping algorithm has higher tumor detection ratio when compared with other brain tumor detection methods. Even though the Skull stripping algorithm has higher segmentation accuracy, due to some traces in the outer region of the skull, this method failed to segment the tumor from all the images.

Baraiya [15] compared the various types of brain tumor extraction methods and its segmentation accuracy. The study involved T2 - Weighted Magnetic Resonance Imaging images. The images were preprocessed and pursued segmentation by using Thresholding, Region-based Segmentation and Watershed Algorithm. The accuracy was around 91.34, 87.48 and 92.76 respectively. The accuracy of these methods varies due to the various threshold values. Moreover, the authors failed to specify the dataset details and the number of images used for evaluating these methods.

Priyanka Kadam [16] presented a method to detect the exact location of a brain tumor and its size in T2 Weighted MRI brain images. By adopting extended maxima transformation, the image was rebuilt to extract tumor, based on various parameters such as length, eccentricity, perimeter, solidity and the region property functions. Though the method is fast and versatile, it was limited to 2D MRI images. As this algorithm's performance was determined by higher intensity and entropy values, the quadrant with less intensity was ignored. Henceforth, there exists a scenario with undetected tumor(s).

Arakeri [17] suggested a content-based image retrieval system (CBIR) in brain tumor diagnosis to take clinical medical decisions. The CBIR system was a bi-folded approach. The first step classified the MRI image and the second step distinguished the image based on different features. To provide faster image retrieval, Principal Component Analysis (PCA) and KD - tree with modified K-Means Clustering Technique were applied. A database of 820 images was experimented to detect Brain 
Tumor. The System yielded a retrieval accuracy of $98.16 \%$ precision and $97.35 \%$ recall.

\section{METHODOLOGY}

Figure 1 shows the flow of the proposed method. Images are obtained from MRI scan. The mentioned scanned pictures are exhibited during $2 \mathrm{D}$ matrices taking pixels as its options. These pictures characteristic features are a grayscale image of size $256 * 256$ pixels. The gray scale image intensities are stretching from 0 to 255 , whereas zero shows the full black color and 255 indicates total white color. The entries amongst these ranges vary from black to white regarding the intensity strength. After the preprocessing stage, Hierarchical Mean Shift algorithm is applied to detect, locate and identify the tumor and its size in the brain MRI.

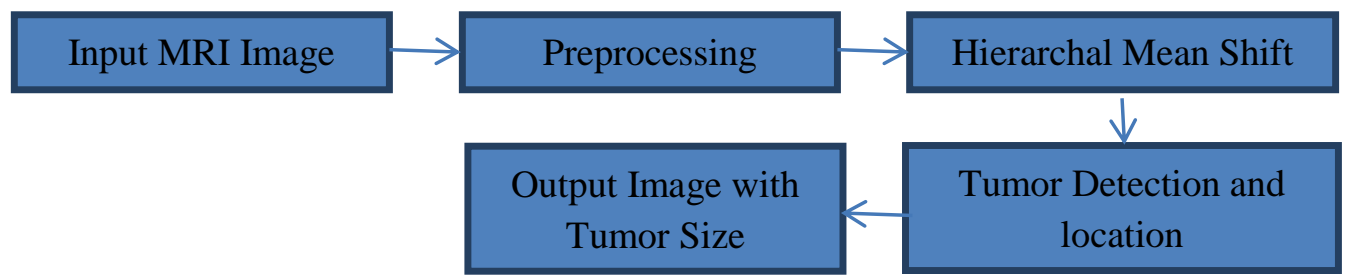

Figure 1 Architecture of the proposed method

\section{(a) Intuitive Idea behind the Hierarchical Mean Shift Algorithm}

The notion of Hierarchical Mean shift was first introduced by Fukunaga and Hostetler [3]. The Hierarchical Mean Shift ponders the feature area as empirical likelihood densities operate function. If the concept could be points, then the Mean shift from the core probability density work is considered. On the opposite part, if the dense cluster areas existent within feature area, then they characterize the native mode as maxima of the likelihood density operate function. Henceforth, the associated groups are realized efficiently and accurately.

For every datum, Hierarchic Mean Shift function is associated with the dataset's neighboring peak of the chance density perform. For each datum, mean shift calculates mean of the information purpose, by process a window around it. Later, it shifts the middle window to the mean and the algorithmic rule is replicated until it convergence and during every iteration, the window shifts from lower denser region to higher thicker region subsequently.

According to [4], Mean shift is defined as,

1. Fixing the window

2. Computing the mean for each such window

3. Shifting the window.

The above steps are repeated until it convergences. 
Some of the preliminaries regarding the kernel to satisfy the requirement are given below:

$$
\int_{R^{d}} \Phi(x)=1
$$

$$
\Phi(x) \geq 0
$$

Some kernel example includes rectangular, Gaussian and Epanechnikov. Parzen window technique, a type of kernel density estimation method, is used to estimate the random variable's density function and is given by the equation (1).

$$
\hat{f}(x)=\frac{1}{n h^{d}} \sum_{i=1}^{n} K\left(\frac{x-x_{i}}{h}\right)
$$

Where, ' $\mathrm{K}$ ' represents the kernel,

' $h$ ' accounts for the bandwidth parameter,

'd' accounts for the Kernel density estimator's dimensional points.

The formula to find the Gradient Ascent of a mean shift is given by the equation (2).

$$
x_{1}=x_{0}+\eta f^{\prime}\left(x_{0}\right)
$$

Applying the kernel density estimator function given in the equation (1) to the Gradient Ascent of a mean shift function (equation 2) and setting to zero, the final result is given by equation (3).

$$
\vec{x}=\frac{\sum_{i=1}^{n} K^{\prime}\left(\frac{x-x_{i}}{h}\right) x_{i}}{\sum_{i=1}^{n} K^{\prime}\left(\frac{x-x_{i}}{h}\right)}
$$

Hierarchical Mean Shift function treats each point of the feature space corresponding to the local maxima or minima as the probability density function. Hence, for each fixed point obtained through gradient ascent represents the density function modes.

Therefore, assuming $\mathrm{g}(\mathrm{x})=-\mathrm{K}^{\prime}(\mathrm{x})$, the equation (3) becomes,

$$
m(x)=\frac{\sum_{i=1}^{n} \mathrm{~g}\left(\frac{x-x_{i}}{h}\right) x_{i}}{\sum_{i=1}^{n} \mathrm{~g}\left(\frac{x-x_{i}}{h}\right)}-x
$$

where, ' $\mathrm{m}(\mathrm{x})$ ' represents the mean shift.

Hence, for each point $\left(\mathrm{x}_{\mathrm{i}}\right)$, the procedure for the mean shift is summarized as:

(1) Computing the average shift vector $\left(m\left(\mathrm{x}_{i}^{t}\right)\right)$

(2) Moving the estimation window by $\left(m\left(\mathrm{x}_{i}^{t}\right)\right)$

(3) Repeat the step (1) and (2) till the convergence reached. 
Mean Shift Algorithm Improvements

The classical algorithm of mean shift is very time-consuming and is given by the equation (5).

$$
O\left(T n^{2}\right)
$$

where, ' $\mathrm{T}$ ' represents the iteration number, and

' $n$ ' represents the data point numbers in the data set.

The Adaptive Mean Shift Algorithm, improvement in the Mean Shift Algorithm, is obtained by varying the bandwidth parameter for each data point. The different bandwidth is given by the equation (6).

$$
\mathrm{h}_{\mathrm{i}}=\left\|x_{i}-x_{i, k}\right\|
$$

where, ' $h$ ' represents the parameter which is calculated using a k-NN algorithm

' $\mathrm{X}_{\mathrm{i}, \mathrm{k}}$ ' represents the k-nearest neighborhood of ' $\mathrm{x}_{\mathrm{i}}$ '

Alternatively, the convergence process is speeded up by altering the data points for the period of Mean Shift procedure.

\section{IMPLEMENTATION}

Initially, the input image is converted into a grayscale image and follows the morphological operations during the preprocessing stage. In the proposed method, Hierarchical Mean Shift algorithm is used to detect and extract the tumor accurately. Resultant of the proposed method is the detection of the tumor in the brain MRI. Further, the advantage of this proposed method is that apart from the identification of tumor in brain MRI, it analyzes the tumor size too.

\section{EXPERIMENTAL RESULTS}

The proposed method is implemented in Matlab version 8.3.0.532 (R2014a) installed on Intel I3 $3.10 \mathrm{GHz}$ processor machine with $2 \mathrm{~GB}$ RAM. To evaluate the proposed method, experiments are carried out on DICOM image sample set [5] database which is exclusively available for the research community. Figure 2 shows the sample MRI brain image with a tumor from the BRAINX dataset [5]. The region shown in dark blue color represents the tumor region. Figure 3 shows the steps to identify the tumor in the brain MRI using the proposed method. The proposed method is assessed by comparing its results with contemporary method [6]. Table 1 shows the comparison of proposed and FCM approach regarding tumor size identification process. Figure 4 shows the comparison chart between the FCM and the proposed method in determining the tumor size in brain MRI. 


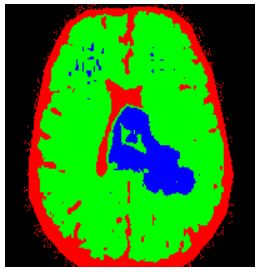

Figure 2. MRI brain image with tumor

\begin{tabular}{|c|c|}
\hline Input image & Identified Tumor Cluster \\
\hline & 0 \\
\hline & \\
\hline
\end{tabular}

Figure 3. Identification of tumor using the proposed system

Table 1. Identification of Tumor Size

\begin{tabular}{|c|c|c|c|}
\hline Sl. No. & Image Number & $\begin{array}{c}\text { Hierarchical Mean } \\
\text { Shift (sq. mm) }\end{array}$ & FCM (sq. mm) \\
\hline 1 & IM-0001-0005.dcm & 1247 & 1179 \\
\hline 2 & IM-0001-0022.dcm & 5174 & 4244 \\
\hline
\end{tabular}
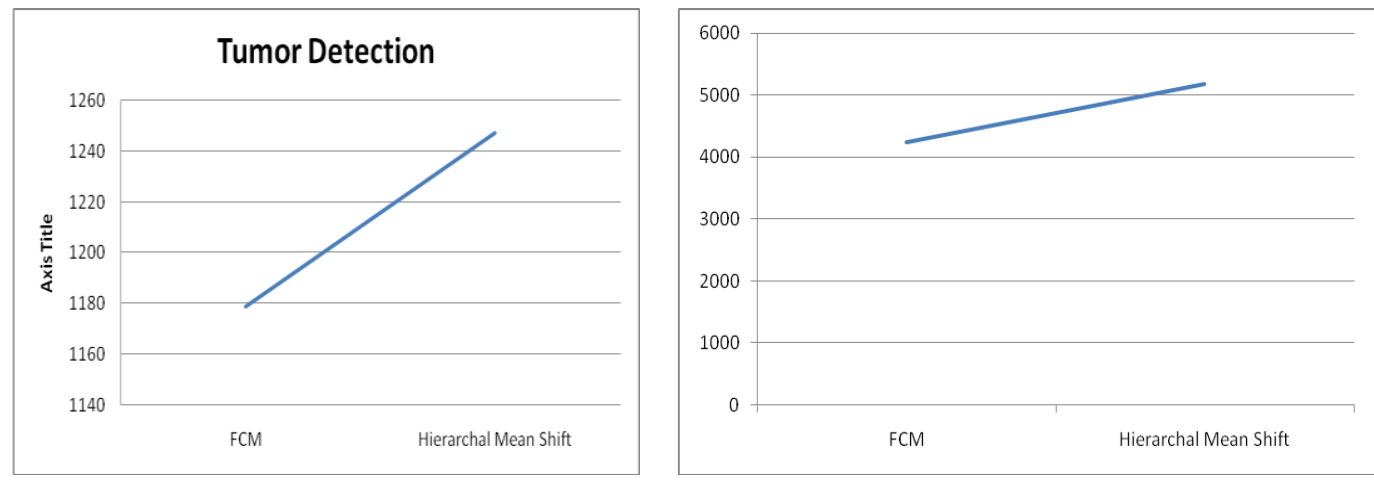

Figure 4. Comparison of proposed method with FCM approach 
Observing the Table 1, it is very clear that the proposed method has detected the exact size of the tumor when compared with the existing FCM approach adopted in [25]. When the proposed method is tested with 22 images from "T1-SE-extrp-601" dataset, the presence of tumor in brain MRI is identified correctly in 20 images. Thus, the segmentation accuracy of the proposed method by using the Hierarchical Mean Shift approach is found to be $90.09 \%$.

\section{CONCLUSION}

The proposed method precisely identified the various regions in the brain MRI, such as white matter, gray matter, cerebrum and finally the tumor with its size regarding square millimeter (sq. mm). Later, the performance of the proposed method was compared with FCM approach. Experimental results concluded that the proposed method had detected the exact tumor size in the brain MRI when compared to FCM approach. As the FCM approach is very sensitive to initialization process, the proposed approach, Hierarchical Mean Shift strategy outperformed the FCM method in detecting the tumor size accurately.

\section{REFERENCES}

[1] Mayer, Arnaldo, and Hayit, G., 2009, "An adaptive mean-shift framework for MRI brain segmentation," IEEE Transactions on Medical Imaging, 28(8), pp. 1238-1250.

[2] Carreira, P., and Miguel, A., 2007, "Gaussian mean-shift is an EM algorithm," IEEE Transactions on Pattern Analysis and Machine Intelligence, 29(5), pp. 767-776.

[3] Fukunaga, and Hostetler, 1975, "The Estimation of the Gradient of a Density Function, with Applications in Pattern Recognition," IEEE Transactions on Information Theory, 21, pp. 32-40.

[4] https://saravananthirumuruganathan.wordpress.com/2010/04/01/introductionto-mean-shift-algorithm/

[5] http://www.osirix-viewer.com/resources/dicom-image-library/

[6] Ganesh, S. R., and Salankar, S. S., 2015, "Brain Tumor Segmentation Using Fuzzy C Means With Ant Colony Optimization Algorithm," Current Trends in Technology and Science, 4(2), pp. 484-490.

[7] JiHoon, K., Sanghun, L., Gang, S. L., Young, S. P., and Yeong, P, H., 2016, "Using a method based on K-Means Clustering and Mean Shift Segmentation to reduce file sizes and detect brain tumors from Magnetic Resonance Images," Wireless Personal Communications, 89(3), pp. 993-1008.

[8] Esmail, H., and Abobakr, A., 2015, "Detecting Brain tumor from MRI image using Matlab GUI programme," International Journal of Computer Science \& Engineering Survey, 6(6), pp. 47-60. 
[9] Pavel, D., Walter, K., and Karel, B., 2013, "Automatic Detection of Brain Tumors in MIR images," $36^{\text {th }}$ International Conference on Telecommunications and Signal Processing (TSP), pp. 577-580.

[10] Shanmugapriya, B., and Ramakrishnan, T., 2014, "Segmentation of Brain Tumors in Computed Tomography images using SVM classifier," International Conference on Electronics and Communication Systems, pp. 1-3.

[11] Ramaraju, P.V., and Shaik, B., 2014, "Brain tumor classification, detection and segmentation using Digital Image Processing and Probabilistic Neural Network Techniques," International Journal of Emerging Trends in Electrical and Electronics, 10(10), pp. 15-20.

[12] Sahar, G., Junning, Li., Ting, L., Paul, S. B., Wendy, D., George, L., 2012, "Automatic Brain tumor detection in Magnetic Resonance Images," $9^{\text {th }}$ IEEE International Symposium on Biomedical Imaging, pp. 572-577.

[13] Ahmed, K., Mohamed, B. M., Nacera, B., Mohamed, A., 2009, "Detection of Brain Tumor in Medical Images," International Conference on Signals, Circuits and Systems, pp. 1-6.

[14] Dimple, K., Kashyap, R., 2016, "Segmentation of Brain Tumor from MRI using Skull Stripping and Neural network," International Journal of Engineering Development and Research, 4(3), pp. 593-598.

[15] Neha, B., and Hardik, M., 2016, "Comparative study of different methods for Brain Tumor Extraction from MRI images using image processing," Indian Journal of Science and Technology, 9(4), pp. 1-5.

[16] Priyanka, K., Pawar, S. N., 2016, "Brain Tumor Segmentation and its feature extraction by using T2 Weighted Brain MRI," International Journal of Advanced Research in Computer and Communication Engineering, 5(7), pp. 166-172.

[17] Megha, P. A., and Reddy, G. R. M., 2013, "An intelligent content-based image retrieval system for clinical decision support in Brain tumor diagnosis," International Journal of Multimedia Information Retrieval, 2, pp. 175-188. 